\title{
UNIVERSAL HOMOTOPY ASSOCIATIVE, HOMOTOPY COMMUTATIVE $H$-SPACES AND THE EHP SPECTRAL SEQUENCE
}

\author{
JELENA GRBIĆ
}

\begin{abstract}
Assume that all spaces and maps are localised at a fixed prime $p$. We study the possibility of generating a universal space $U(X)$ from a space $X$ which is universal in the category of homotopy associative, homotopy commutative $H$-spaces in the sense that any map $f: X \longrightarrow Y$ to a homotopy associative, homotopy commutative $H$-space extends to a uniquely determined $H$-map $\bar{f}: U(X) \longrightarrow Y$. Developing a method for recognising certain universal spaces, we show the existence of the universal space $F_{2}(n)$ of a certain three-cell complex $L$. Using this specific example, we derive some consequences for the calculation of the unstable homotopy groups of spheres, namely, we obtain a formula for the $d_{1}$-differential of the $E H P$-spectral sequence valid in a certain range.
\end{abstract}

\section{INTRODUCTION}

In this paper we investigate the possibility of generating an $H$-space out of its subspace and derive some consequences for the calculations of the unstable homotopy groups of spheres. Particular emphasis is laid on finding a formula for the first differential $d_{1}$ in the EHP spectral sequence which is valid in a certain range.

Universal objects have always been of great interest to mathematicians in different mathematical disciplines. Specifically, in homotopy theory one of the first universal spaces is given in the category of homotopy associative $H$-spaces by the James construction. Namely, if $X$ is a topological space with a non-degenerate basepoint $e$ and a compactly generated topology, then the James construction $J(X)$ on $X$ is the free topological monoid generated by $X$ subject to the single relation that the basepoint $e$ is the unit. The James construction satisfies the following universal property: if $Y$ is a homotopy associative $H$-space and a map $f: X \longrightarrow Y$ is given, then $f$ extends to an $H$-map $\tilde{f}: J(X) \longrightarrow Y$ which is unique up to homotopy.

Our aim is to find further information concerning universal spaces. Indeed, one can ask what happens when the category of homotopy 
associative $H$-spaces is replaced with the category of homotopy associative, homotopy commutative $H$-spaces. Do universal spaces exist in this category and if they do, is there a general construction of them? While we do not answer the above questions in full generality, we do present a new method for constructing universal spaces, and describe some new universal spaces and their applications in homotopy theory. For the sake of completeness we include all the details as well as a brief overview of known examples of universal spaces.

The method and its applications are $p$-local, so throughout the remainder of the paper all spaces and maps have been localised at a prime $p$. Also we assume that all spaces are pointed, connected topological spaces with the homotopy types of finite type $C W$-complexes. Unless otherwise indicated, the ring of homology coefficients will be $\mathbb{Z} / p \mathbb{Z}$ and $H_{*}(X ; \mathbb{Z} / p \mathbb{Z})$ will be written as $H_{*}(X)$.

In particular, aiming for a better understanding of the double loop space on an odd sphere, we consider the first few spaces appearing in Selick's filtration [14] of $\Omega^{2} S^{2 n+1}$. The main object of concern is a space $F_{2}(n)$ which is defined as the homotopy pullback

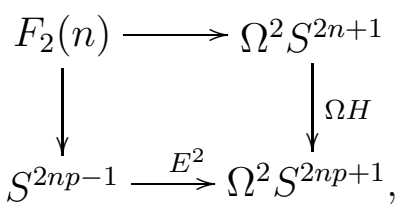

where $H: \Omega S^{2 n+1} \longrightarrow \Omega S^{2 n p+1}$ is the James-Hopf invariant map and $E^{2}$ is the double suspension.

Let $L$ denote the $(2 n p-1)$-skeleton of $F_{2}(n)$. The central result of the paper is as follows.

thm[A] The space $F_{2}(n)$ is the universal space of $L$ in the category of homotopy associative, homotopy commutative $H$-spaces. thm By $F_{2}(n)$ being a universal space in the category of homotopy associative, homotopy commutative $H$-spaces, we mean that $F_{2}(n)$ has the two properties:

(i) the space $F_{2}(n)$ is a homotopy associative and homotopy commutative $H$-space;

(ii) let $Y$ be a homotopy associative, homotopy commutative $H$ space and $f: L \longrightarrow Y$ a given map. Then there exists an $H$-map $\bar{f}: F_{2}(n) \longrightarrow Y$ extending $f$, and it is a unique up to homotopy,.

The universal property of $F_{2}(n)$ can be used to obtain useful information about the $d_{1}$-differential in the EHP spectral sequence (cf. [13]). This spectral sequence is used to calculate the homotopy groups of 
spheres (cf. [19]), and is made up by interlocking the classical EHP sequences for various spheres together. These $E H P$ sequences are long exact sequences of the homotopy groups of spheres induced by the two classical homotopy fibrations:

$$
\Omega^{2} S^{2 n p+1} \stackrel{P}{\longrightarrow} J_{p-1}\left(S^{2 n}\right) \stackrel{E}{\longrightarrow} \Omega S^{2 n+1} \stackrel{H}{\longrightarrow} \Omega S^{2 n p+1}
$$

and

$$
\Omega^{2} S^{2 n p-1} \stackrel{P}{\longrightarrow} S^{2 n-1} \stackrel{E}{\longrightarrow} \Omega J_{p-1}\left(S^{2 n}\right) \stackrel{H}{\longrightarrow} \Omega S^{2 n p-1},
$$

where $J_{k}(X)$ is the $k^{t h}$-stage of the James construction on a topological space $X$, and the map $H: \Omega J_{p-1}\left(S^{2 n}\right) \longrightarrow \Omega S^{2 n p-1}$ is the TodaHopf invariant map. In the literature the Toda-Hopf map is sometimes denoted by $T$ to distinguish it more explicitly from the James-Hopf invariant map.

Namely, for $p>2$ there are spectral sequences with $E_{1}^{k, 2 n+1}=$ $\pi_{k+2 n+1}\left(S^{2 n p+1}\right)$ and $E_{1}^{k, 2 n}=\pi_{k+2 n}\left(S^{2 n p-1}\right)$. The spectral sequence with $E^{k, n}=0$ for $n>j$ converges to $\pi_{*}\left(S^{j}\right)$ if $j$ is odd and to $\pi_{*}\left(J_{p-1}\left(S^{j}\right)\right)$ if $j$ is even (cf.[13]).

A key calculation in the EHP spectral sequence is that of the first differential $d_{1}: \pi_{r+2}\left(S^{2 n p+1}\right) \longrightarrow \pi_{r}\left(S^{2 n p-1}\right)$. The $d_{1}$-differential is induced by the composition $\Omega^{3} S^{2 n p+1} \stackrel{\Omega P}{\longrightarrow} \Omega J_{p-1}\left(S^{2 n}\right) \stackrel{H}{\longrightarrow} \Omega S^{2 n p-1}$. In the metastable range, the $d_{1}$-differential is completely determined by Toda's formula [19]: $d_{1}\left(E^{2} x\right)=p \cdot x$. In [8] Gray constructed a map $\varphi_{n}: \Omega^{2} S^{2 n p+1} \longrightarrow S^{2 n p-1}$ with the property that $\Omega \varphi_{n}=H \circ \Omega P$. Using the existence of the map $\varphi_{n}$, Gray [9] developed a formula for the $d_{1}$-differential applicable to some elements which are not double suspensions. In [9] Gray showed that $\Omega J_{p-1}\left(S^{2 n}\right)$ for $p>2$ is universal in the category of homotopy associative, homotopy commutative $H$-spaces, with its generating subspace being the $(2 n p-2)$-skeleton. He used this to extend the formula involving $d_{1}$-differential. We shall extend further Gray's result using the universality of $F_{2}(n)$.

Notation 1.1. In the case of odd spheres localised at a prime $p \geq 3$, the degree $p$ map and the $p$-power map are homotopic and are commonly denoted by $p: S^{2 n+1} \longrightarrow S^{2 n+1}$. Denote by $S^{2 n+1}\{p\}$ the homotopy fibre of the degree $p$ map on $S^{2 n+1}$. The homotopy cofibre of the degree $p^{r}$ map on $S^{n-1}$ for $n \geq 2$ and $r \geq 1$ is called the $n$-dimensional p-primary Moore space and is denoted by $P^{n}\left(p^{r}\right)$. For any topological space $X$, the adjoint of the identity map on $\Sigma X$ gives a map $E: X \longrightarrow \Omega \Sigma X$, usually called the suspension map. The double suspension $E^{2}: S^{2 n-1} \longrightarrow \Omega^{2} S^{2 n+1}$ is the double adjoint of the identity map on $S^{2 n+1}$. Its homotopy fibre is denoted by $W_{n}$. In [8] Gray has shown that $W_{n}$ has a classifying space $B W_{n}$. 
Using the definition of $F_{2}(n)$ as the homotopy pullback in diagram (11), there exist two fibration sequences analogous to the classical EHP fibrations. pro[B] There exist two homotopy fibration sequences:

$$
W_{n p} \stackrel{P}{\longrightarrow} F_{2}(n) \stackrel{E}{\longrightarrow} \Omega^{2} S^{2 n+1} \stackrel{H}{\longrightarrow} B W_{n p}
$$

and

$$
\Omega S^{2 n p-1}\{p\} \stackrel{P}{\longrightarrow} S^{2 n-1} \stackrel{E}{\longrightarrow} F_{2}(n) \stackrel{H}{\longrightarrow} S^{2 n p-1}\{p\} .
$$

pro Weaving together the long exact sequences of homotopy groups induced by the above fibration sequences, we obtain an analogous EHP spectral sequence to the classical one.

For $p>2$ there are spectral sequences with $E_{1}^{k, 2 n+1}=\pi_{k+2 n+1}\left(W_{n p}\right)$ and $E_{1}^{k, 2 n}=\pi_{k+2 n}\left(S^{2 n p-1}\{p\}\right)$. The spectral sequence with $E^{k, n}=0$ for $n>j$ converges to $\pi_{*}\left(S^{j}\right)$ if $j$ is odd and to $\pi_{*}\left(F_{2}\left(\frac{j}{2}\right)\right)$ if $j$ is even.

Using the universality of $F_{2}(n)$, we obtain the following formula involving the $d_{1}$-differential. thm $[\mathrm{C}]$ The composition

$$
F_{2}(n p) \stackrel{E}{\longrightarrow} \Omega^{2} S^{2 n p+1} \stackrel{\varphi_{n}}{\longrightarrow} S^{2 n p-1} \stackrel{E}{\longrightarrow} F_{2}(n p)
$$

is the $p$-power map if either

(i) $x \in \pi_{*}\left(F_{2}(n p)\right)$ is an element which is a lift through $H: F_{2}(n p) \longrightarrow S^{2 n p^{2}-1}\{p\}$ of an element in the image of $E: P^{2 n p^{2}-2}(p) \longrightarrow \Omega S^{2 n p^{2}-1}\{p\}$

or

(ii) the composition $E \circ \varphi_{n} \circ E$ is an $H$-map. thm

cor[D] Passing to homotopy groups under either assumption (i) or assumption (ii) of Theorem $\mathrm{C}$, the formula for the $d_{1}$-differential takes the form

$$
E d_{1}(E x)=p \cdot x
$$

extending Gray's analogous result [9] with respect to $\Omega J_{p-1}\left(S^{2 n}\right)$. cor

Theorem C (ii) brings up once more the question whether $\varphi_{n}$ is an $H$-map. This has not been proved as yet. As a first step in that direction we have the following result.

pro[E] Restricted to the $\left(2 n p^{3}-4\right)$-skeleton the composite

$$
F_{2}(n p) \longrightarrow \Omega^{2} S^{2 n p+1} \stackrel{\varphi_{n}}{\longrightarrow} S^{2 n p-1} \stackrel{E}{\longrightarrow} F_{2}(n p)
$$

is homotopic to the $p$-power map restricted to $\left(F_{2}(n p)\right)_{\left(2 n p^{3}-4\right)}$. pro Theorem C and Proposition E imply that formula (2) for the $d_{1}$ differential is valid up to the $\left(2 n p^{3}-4\right)$-skeleton of $F_{2}(n p)$. Since the 
$\left(2 n p^{3}-4\right)$-skeleton of $F_{2}(n p)$ is homotopy equivalent to the $\left(2 n p^{3}-4\right)$ skeleton of $\Omega^{2} S^{2 n p+1}$, formula (2) determines the composite of the $d_{1}$ differential with $E: S^{2 n p-1} \longrightarrow F_{2}(n p)$ restricted to the $\left(2 n p^{3}-4\right)$ skeleton of $\Omega^{2} S^{2 n p+1}$.

This improves Gray's result in the sense that formula (21) is not anymore valid just for $\left(2 n p^{2}-2\right)$-skeleton but for $\left(2 n p^{3}-4\right)$-skeleton of $\Omega^{2} S^{2 n p+1}$.

\section{UNIVERSAL HOMOTOPY ASSOCIATIVE, HOMOTOPY COMMUTATIVE $H$-SPACES}

2.1. Preliminary definitions and known results. This section deals with the concept of universal spaces, sometimes called generated spaces. It sets up a new approach for constructing universal spaces in the category of homotopy associative, homotopy commutative $H$-spaces.

Definition 2.1. Let $X$ be a space, $G(X)$ an $H$-space and $f: X \longrightarrow G(X)$ a continuous map. $(X, f)$ will be called a generator for $G(X)$ if and only if the following universal property is satisfied: for each $H$-space $Y$, the mapping

$$
\{\text { homotopy classes of } H \text { maps } G(X) \rightarrow Y\} \stackrel{f^{*}}{\longrightarrow}[X, Y]
$$

is a bijection.

Remark 2.2. A space $G(X)$ satisfying the stated universal property is called the universal space of $X$. Looking at the universal property it is easy to notice that $G(X)$ is unique.

One of the main examples of generated spaces is provided by the James construction, which we described in the Introduction. In the sense of Definition 2.1, the James construction has the following universal property: if $Y$ is any homotopy associative $H$-space and $f: X \longrightarrow Y$ is any continuous map, then $f$ has an extension to a unique $H$-map $\tilde{f}: J(X) \longrightarrow Y$. We call $\tilde{f}$ the multiplicative extension of $f$. In particular, if $X$ is path connected, then the suspension map $E: X \longrightarrow \Omega \Sigma X$ extends to a multiplicative homotopy equivalence $\widetilde{E}: J(X) \longrightarrow \Omega \Sigma X$. Therefore, the James construction on $X$ can be regarded as a combinatorial model for $\Omega \Sigma X$. In this paper we identify $J(X)$ with $\Omega \Sigma X$.

We can slightly modify the definition of the generating complex asking for both spaces $G(X)$ and $Y$ to belong to the category of homotopy associative, homotopy commutative $H$-spaces. With this additional condition Definition 2.1 can be reformulated as follows.

Definition 2.3. A universal space $U(X)$ of a space $X$ is a homotopy associative, homotopy commutative $H$-space (localised at $p$ ) together 
with a map $i: X \longrightarrow U(X)$ such that the following universal property holds:

if $Y$ is a homotopy associative, homotopy commutative $H$-space (localised at $p$ ) and $f: X \longrightarrow Y$ is any map, then $f$ extends to a unique $H$-map $\bar{f}: U(X) \longrightarrow Y$.

Observe that the mapping induced by the definition of universal spaces

$$
\{\text { homotopy classes of } H \text { maps } U(X) \rightarrow Y\} \longrightarrow[X, Y]
$$

is a bijection.

Remark 2.4. Notice that the universal space $U(X)$ in the category of homotopy associative, homotopy commutative $H$ spaces is defined by a space localised at a prime $p$ rather then by an integral, non-localised, space. The only reason for considering a localised version of universal spaces is the existences of a better developed machinery that can be applied to $p$-localised spaces in order to describe their properties.

Known examples of universal spaces in the category of homotopy associative, homotopy commutative $H$-spaces. 1 . The simplest example of universal spaces appears when we look for a universal space of a torsion free space, that is, of a rational space. The universal space of a rational space $X$ always exists and is given by the infinite symmetric product $S P^{\infty}(X)$. This fact was proved by Dold and Thom [7, although they did not use the terminology of universal spaces.

2. The fact that some examples of $U(X)$ exist, even with the additional homology condition, can easily be seen in the case of spheres. The universal space $U\left(S^{2 n}\right)$ of an even dimensional sphere for $p \geq 3$ is already given by the James construction on $S^{2 n}$ since $\Omega S^{2 n+1}$ is homotopy commutative as well as homotopy associative [1]. The universal space $U\left(S^{2 n-1}\right)$ of an odd dimensional sphere is almost implicitly given by Serre's splitting [15] at odd primes of the loop space on an even dimensional sphere $\Omega S^{2 n} \stackrel{\simeq}{\longrightarrow} S^{2 n-1} \times \Omega S^{4 n-1}$. The equivalence is obtained via the product of the map $E: S^{2 n-1} \longrightarrow \Omega S^{2 n}$ with the loop map on the Whitehead product $\omega: S^{4 n-1} \longrightarrow S^{2 n}$ of the identity map on $S^{2 n}$ with itself. For $p \geq 5$, an odd dimensional sphere $S^{2 n-1}$ is homotopy associative, homotopy commutative, and $U\left(S^{2 n-1}\right)=S^{2 n-1}$. A more detailed proof for the universal spaces of spheres reader can find in [11].

3. The next example of universal spaces occurs in the work of Cohen, Moore and Neisendorfer [5] on splitting the loop spaces on even dimensional odd primary Moore spaces $\Omega P^{2 n+2}\left(p^{r}\right) \stackrel{\simeq}{\longrightarrow} S^{2 n+1}\left\{p^{r}\right\} \times \Omega\left(\vee_{\alpha} P^{n_{\alpha}}\left(p^{r}\right)\right)$. 
In this case, Gray showed 9] that $U\left(P^{2 n+1}\left(p^{r}\right)\right)=S^{2 n+1}\left\{p^{r}\right\}$, the homotopy fibre of the degree $p^{r}$ map on $S^{2 n+1}$.

4. The most difficult known examples of $U(X)$ in the sense that they do not retract off the James construction $J(X)$ on $X$ are those $H$ spaces whose homology rings are the abelianisation of the homology ring of the loop space on odd dimensional Moore spaces at odd prime. These spaces have been constructed by Anick and Gray [2, 3] and their universality has been shown by Theriault [16. In this case the universal space of $P^{2 n}\left(p^{r}\right)$ does not split off the James construction on $P^{2 n}\left(p^{r}\right)$ unlike the other cases above.

5. An example of a universal space that is closely related to the universal space we are interested in considers a loop space $\Omega J_{p-1}\left(S^{2 n}\right)$. Let $K_{n}$ be the $(2 n p-2)$-skeleton of $\Omega J_{p-1}\left(S^{2 n}\right)$. Gray [9] has shown that $\Omega J_{p-1}\left(S^{2 n}\right)$ is homotopy commutative as well as homotopy associative, due to the loop space structure, and that it is the universal space of $K_{n}$.

\subsection{A homological filtration of the double suspension of odd}

dimensional spheres. In order to construct a spectral sequence concerning the double suspension Selick 14 looked at a certain filtration of $\Omega^{2} S^{2 n+1}$. We recall it here briefly. Looping the filtration

$$
\{p t\} \longrightarrow J_{1}\left(S^{2 n}\right) \longrightarrow \ldots \longrightarrow J_{k}\left(S^{2 n}\right) \longrightarrow \ldots \longrightarrow J\left(S^{2 n}\right) \simeq \Omega \Sigma S^{2 n}
$$

of the James construction on $S^{2 n}$ gives a filtration

$$
\{p t\} \longrightarrow \Omega J_{1}\left(S^{2 n}\right) \longrightarrow \ldots \longrightarrow \Omega J_{k}\left(S^{2 n}\right) \longrightarrow \ldots \longrightarrow \Omega^{2} S^{2 n+1}
$$

of $\Omega^{2} S^{2 n+1}$.

It is well known (cf. 4]) that

$$
H_{*}\left(\Omega^{2} S^{2 n+1}\right) \cong \bigotimes_{j=0}^{\infty} \Lambda\left(x_{2 n p^{j}-1}\right) \otimes \bigotimes_{j=1}^{\infty} \mathbb{Z} / p \mathbb{Z}\left[y_{2 n p^{j}-2}\right]
$$

with $\beta x_{2 n p^{j}-1}=y_{2 n p^{j}-2}$. Applying the Serre or Eilenberg-Moore spectral sequence to the homotopy fibration sequence

$$
\Omega J_{p^{k-1}}\left(S^{2 n}\right) \longrightarrow \Omega^{2} S^{2 n+1} \longrightarrow \Omega^{2} S^{2 n p^{k}+1},
$$

it follows that

$$
H_{*}\left(\Omega J_{p^{k}-1}\left(S^{2 n}\right)\right) \cong \bigotimes_{j=0}^{k-1} \Lambda\left(x_{2 n p^{j}-1}\right) \otimes \bigotimes_{j=1}^{k} \mathbb{Z} / p \mathbb{Z}\left[y_{2 n p^{j}-2}\right]
$$

Therefore, the filtration

$$
\{p t\} \longrightarrow \Omega J_{p-1}\left(S^{2 n}\right) \longrightarrow \ldots \longrightarrow \Omega J_{p^{k-1}}\left(S^{2 n}\right) \longrightarrow \ldots \longrightarrow \Omega^{2} S^{2 n+1}
$$


gives a natural filtration of $H_{*}\left(\Omega^{2} S^{2 n+1}\right)$. The aim is to obtain a full homological filtration of $\Omega^{2} S^{2 n+1}$. To refine filtration (3) we shall insert spaces, denoted by $F_{2 k}(n)$, between $\Omega J_{p^{k-1}}\left(S^{2 n}\right)$ and $\Omega J_{p^{k+1}-1}\left(S^{2 n}\right)$ requiring that their homology is

$$
H_{*}\left(F_{2 k}(n)\right) \cong \bigotimes_{j=0}^{k} \Lambda\left(x_{2 n p^{j}-1}\right) \otimes \bigotimes_{j=1}^{k} \mathbb{Z} / p \mathbb{Z}\left[y_{2 n p^{j}-2}\right] .
$$

Selick's filtration

$$
F_{-1}(n) \longrightarrow F_{0}(n) \longrightarrow F_{1}(n) \rightarrow \ldots \rightarrow F_{k}(n) \longrightarrow \ldots \longrightarrow F_{\infty}(n)
$$

is defined inductively on $n$ and $k$. Set the initial data in a way that $F_{-1}(n)=\{p t\}, F_{0}(n)=S^{2 n-1}$ and $F_{\infty}(n)=\Omega^{2} S^{2 n+1}$. Assume by induction on $k$ that for all $q \leq k$ and for all $n$ the spaces $F_{q}(n)$ have been constructed along with maps

$$
F_{-1}(n) \longrightarrow F_{0}(n) \longrightarrow F_{1}(n) \longrightarrow \ldots \longrightarrow F_{k}(n) \longrightarrow F_{\infty}(n) .
$$

The space $F_{k+1}(n)$ is defined as the homotopy pullback

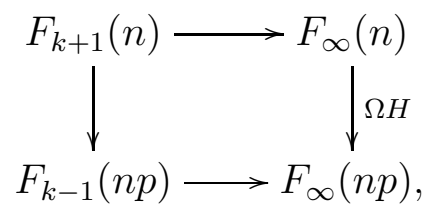

where $H: \Omega S^{2 n+1} \longrightarrow \Omega S^{2 n p+1}$ is the James-Hopf invariant map. Being a homotopy pullback of $H$-spaces and $H$-maps each $F_{k}(n)$ is an $H$-space and all the induced maps in the pullback diagram are $H$-maps. Toda's calculations [18] show that $F_{2 k-1}(n) \simeq \Omega J_{p^{k-1}}\left(S^{2 n}\right)$. Therefore, we conclude that in filtration (41) all the spaces are $H$-spaces and all the maps are $H$-maps.

In particular, $F_{2}(n)$ is defined as the homotopy pullback

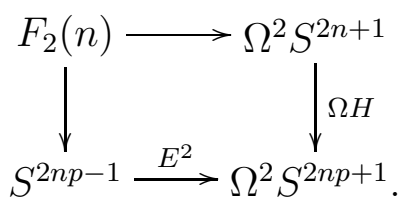

In this way, $F_{2}(n)$ can be considered as the third stage in an approximation of $\Omega^{2} S^{2 n+1}$.

Therefore, the knowledge of $F_{2}(n)$ can be used to better understand the map $\varphi_{n}: \Omega^{2} S^{2 n p+1} \longrightarrow S^{2 n p-1}$, discussed in the Introduction, and to extend the calculations of the $d_{1}$-differential in the EHP spectral sequence. 
2.3. The strategy for the proof of Theorems B and C. In this short section we outline the strategy for the proof of Theorems B and $\mathrm{C}$ in order to facilitate the reading and the understanding of the main ideas. We briefly give the major facts and procedures.

Consider the homology of $F_{2}(n)$, that is,

$$
H_{*}\left(F_{2}(n)\right) \cong \Lambda\left(x_{2 n-1}, x_{2 n p-1}\right) \otimes \mathbb{Z} / p \mathbb{Z}\left[y_{2 n p-2}\right],
$$

the symmetric algebra on three generators $x_{2 n-1}, y_{2 n p-2}, x_{2 n p-1}$.

Denote by $K$ the $(2 n p-2)$-skeleton of $\Omega J_{p-1}\left(S^{2 n}\right)$. Then $K$ maps to $F_{2}(n)$ by the composition

$$
K \longrightarrow \Omega J_{p-1}\left(S^{2 n}\right)=F_{1}(n) \longrightarrow F_{2}(n)
$$

implying that the pair $\left(F_{2}(n), K\right)$ is $2 n p-2$ connected. Let

$$
(h, q):\left(e^{2 n p-1}, S^{2 n p-2}\right) \longrightarrow\left(F_{2}(n), K\right)
$$

represent a generator of

$$
\pi_{2 n p-1}\left(F_{2}(n), K\right) \cong H_{2 n p-1}\left(F_{2}(n), K ; \mathbb{Z}\right) .
$$

Define a space $L$ as the homotopy cofibre of $q$, namely, as the three-cell complex $L=K \cup_{q} e^{2 n p-1}$. Hence $L$ is the $(2 n p-1)$-skeleton of $F_{2}(n)$, where each cell corresponds to a generator of $S\left(x_{2 n-1}, y_{2 n p-2}, x_{2 n p-1}\right)$.

The general idea in constructing the universal space of $L$ is to look first at the James construction on $L$. Since $\Omega \Sigma L$ is not homotopy commutative (if it were, its homology would be a symmetric algebra) it cannot be the universal space of $L$. Therefore, for a suitable candidate for the universal space we aim for a homotopy associative, homotopy commutative retract of $\Omega \Sigma L$ containing $L$ as a subspace. Since the universal space of $L$ is by definition homotopy commutative, its homology is commutative, so an initial guess for the homology ring would be the abelianisation of $T\left(\widetilde{H}_{*}(L)\right)$, that is the symmetric algebra $S\left(\widetilde{H}_{*}(L)\right)$. Our method for constructing the universal space of $L$ involves three steps. In the first two steps we are constructing a suitable retract of $\Omega \Sigma L$. We start by obtaining a decomposition of $H_{*}(\Omega \Sigma L)$, where one of the factors is a symmetric algebra. To do this we regard $H_{*}(\Omega \Sigma L)$ as a universal enveloping algebra and apply the theory of Lie algebras.

Lemma 2.1. There is an isomorphism

$$
H_{*}(\Omega \Sigma L) \cong T(W) \otimes S\left(x_{2 n-1}, y_{2 n p-2}, x_{2 n p-1}\right)
$$

of left $T(W)$-modules and right $S\left(x_{2 n-1}, y_{2 n p-2}, x_{2 n p-1}\right)$-comodules, where $W$ is a free $\mathbb{Z} / p \mathbb{Z}$-module. 
The construction is then based on a geometric realisation of the homological decomposition, where each factor is realised as a space of a certain principal fibration.

Proposition 2.2. There is a fibration sequence

$$
\Omega \Sigma R \stackrel{\Omega \omega}{\longrightarrow} \Omega \Sigma L \longrightarrow F_{2}(n) \longrightarrow \Sigma R \stackrel{\omega}{\longrightarrow} \Sigma L,
$$

where $\Sigma R$ is a wedge of spheres and Moore spaces, and $\omega$ is a sum of Whitehead products and mod $p$ Whitehead products. Moreover, $\Omega \omega$ has a left homotopy inverse.

Corollary 2.3. There is a homotopy decomposition

$$
\Omega \Sigma L \simeq F_{2}(n) \times \Omega \Sigma R .
$$

If Proposition 2.2 is granted, then (5) shows that $F_{2}(n)$ corresponds to a geometric realisation of the symmetric algebra $S\left(x_{2 n-1}, y_{2 n p-2}, x_{2 n p-1}\right)$. In the third and last step we shall further examine fibration (66) in order to prove that $F_{2}(n)$ is the universal space space of $L$ in the category of homotopy associative, homotopy commutative $H$-spaces, that is, we shall prove Theorems B and C.

2.4. The construction of a map $\omega: \Sigma R \longrightarrow \Sigma L$. We want to construct a map $\omega$ which takes a certain wedge of spheres and Moore spaces $\Sigma R$ into $\Sigma L$ by means of Whitehead products and mod $p$ Whitehead products in such a way that the homology map $(\Omega \omega)_{*}$ is an isomorphism onto the subalgebra generated by Lie brackets in $H_{*}(\Omega \Sigma L)$. Therefore, $\omega$ realises a decomposition of the homology of $\Omega \Sigma L$ given as a product of $H_{*}(\Omega \Sigma R)$ and some symmetric algebra.

A purely algebraic approach gives prominent insight into this problem. The following analysis has been drawn from the work of Cohen, Moore and Neisendorfer [5].

Notation 2.5. Throughout this section, let $R$ be a commutative ring in which 2 is a unit. If $V$ is positively graded module over $R$, then $\mathcal{L}\langle V\rangle$ is the free graded Lie algebra over $R$ generated by $V$. In particular, $\mathcal{L}=\mathcal{L}\langle x, u, v\rangle$ is the Lie algebra over $R$ on three generators $x, u, v$ in degrees $2 n-1,2 n p-2$, and $2 n p-1$, respectively. Denote by $[\mathcal{L}, \mathcal{L}]$ the commutator of $\mathcal{L}$. We regard $\mathcal{L}_{a b}\langle V\rangle$ as the free graded abelian Lie algebra over $R$ generated by $V$. The universal enveloping algebra of $\mathcal{L}$ is denoted by $U \mathcal{L}$. The universal enveloping algebra $U \mathcal{L}\langle V\rangle$ of the free Lie algebra $\mathcal{L}\langle V\rangle$ is isomorphic to the free tensor algebra $T(V)$ generated by $V$, and hence $U \mathcal{L}_{a b}\langle V\rangle$ is isomorphic to the free symmetric algebra $S(V)$ generated by $V$. 
By the definition of a free graded abelian Lie algebra there is a short exact sequence of Lie algebras

$$
0 \longrightarrow[\mathcal{L}, \mathcal{L}] \longrightarrow \mathcal{L} \longrightarrow \mathcal{L}_{a b}\langle x, u, v\rangle \longrightarrow 0 .
$$

According to [5, Proposition 3.7], this results in a short exact sequence of homology Hopf algebras

$$
0 \longrightarrow U[\mathcal{L}, \mathcal{L}] \longrightarrow U \mathcal{L} \longrightarrow U \mathcal{L}_{a b}\langle x, u, v\rangle \longrightarrow 0,
$$

and an isomorphism

$$
U \mathcal{L} \cong U[\mathcal{L}, \mathcal{L}] \otimes U \mathcal{L}_{a b}\langle x, u, v\rangle
$$

of left $U[\mathcal{L}, \mathcal{L}]$-modules and right $U \mathcal{L}_{a b}\langle x, u, v\rangle$-comodules. On the other hand, whenever $\mathcal{L}$ is a connected free Lie algebra its commutator $[\mathcal{L}, \mathcal{L}]$, as a sub-Lie algebra of $\mathcal{L}$, is a free Lie algebra $\mathcal{L}\langle W\rangle$ with $W$ a free $R$-module. Therefore it follows that

$$
U[\mathcal{L}, \mathcal{L}] \cong T(W) .
$$

Proof of Lemma 2.1. Notice that $H_{*}(L)$ is the free $\mathbb{Z} / p \mathbb{Z}$-module with basis $x, u$ and $v$ in degrees $2 n-1,2 n p-2$ and $2 n p-1$. Since $H_{*}(L)$ is a trivial coalgebra, $H_{*}(\Omega \Sigma L)$ is the primitively generated tensor Hopf algebra $T\left(x_{2 n-1}, u_{2 n p-2}, v_{2 n p-1}\right)$ generated by $x, u$ and $v$. Therefore $H_{*}(\Omega \Sigma L)$ can also be considered as the universal enveloping Hopf algebra $U \mathcal{L}$ of the free Lie algebra $\mathcal{L}=\mathcal{L}\langle x, u, v\rangle$.

Applying decomposition (77) of the universal enveloping algebra $H_{*}(\Omega \Sigma L)$, we get an isomorphism

$$
H_{*}(\Omega \Sigma L) \cong T(W) \otimes S\left(x_{2 n-1}, u_{2 n p-2}, v_{2 n p-1}\right),
$$

of left $T(W)$-modules and right $S\left(x_{2 n-1}, u_{2 n p-2}, v_{2 n p-1}\right)$-comodules, where $W$ is a free $\mathbb{Z} / p \mathbb{Z}$-module. This proves Lemma 2.1.

We would like to have better control over the free $\mathbb{Z} / p \mathbb{Z}$-module $W$. Recall that the Euler-Poincaré series of a free graded module $V=$ $\bigoplus_{n=0}^{\infty} V_{n}$ of finite type is the power series

$$
\chi(V)=\sum_{n=0}^{\infty}\left(\operatorname{rank} V_{n}\right) t^{n} .
$$

Suppose that $\mathcal{L}^{\prime} \longrightarrow \mathcal{L} \longrightarrow \mathcal{L}^{\prime \prime}$ is a short exact sequence of connected graded Lie algebras which are free finite type $R$-modules. If $\mathcal{L}$ is a free Lie algebra $\mathcal{L}\langle V\rangle$, then by [5. Corollary 3.11] $\mathcal{L}^{\prime}$ is a free Lie algebra $\mathcal{L}\langle W\rangle$, where $W$ is a free finite type $R$-module with the Euler-Poincaré series given by

$$
\chi(W)=1-\chi\left(\mathcal{U L}^{\prime \prime}\right)(1-\chi(V)) .
$$


In our case $\mathcal{L}$ is the free Lie algebra on three generators $x_{2 n-1}, u_{2 n p-2}$ and $v_{2 n p-1}$, and we have written the commutator $[\mathcal{L}, \mathcal{L}]$ as the free Lie algebra $\mathcal{L}(W)$ generated by a finite-type $\mathbb{Z} / p \mathbb{Z}$-module $W$.

Lemma 2.4. The Euler-Poincaré series of $[\mathcal{L}, \mathcal{L}]$ has the following form

$$
\begin{aligned}
\chi(W)=\left(t^{4 n-2}+t^{2 n(p+1)-3}+t^{2 n(p+1)-2}+t^{2 n(p+2)-3}+\right. \\
\left.\quad t^{4 n p-3}+t^{4 n p-2}+t^{2 n(2 p+1)-4}+t^{2 n(2 p+1)-3}\right) \sum_{k=0}^{\infty} t^{2 k(n p-1)} .
\end{aligned}
$$

Proof. The Euler-Poincaré series for $W$ is given by equation (8), namely,

$$
1-\chi(W)=\chi\left(\mathcal{U} \mathcal{L}_{a b}\langle x, u, v\rangle\right)(1-\chi(x, u, v)) .
$$

Expanding the right side of equation (9) gives

$$
1-\chi(W)=\frac{\left(1+t^{2 n-1}\right)\left(1+t^{2 n p-1}\right)}{1-t^{2 n p-2}}\left(1-t^{2 n-1}-t^{2 n p-2}-t^{2 n p-1}\right) .
$$

Sorting out the previous expression the stated formula for $\chi(W)$ is obtained.

Remark 2.6. For $x, y \in \mathcal{L}$, the Lie bracket $[x, y]$ in $\mathcal{L}$ is also denoted by $\operatorname{ad}(x)(y)$. This notation can be generalised by defining $\operatorname{ad}^{0}(x)(y)=y$ and for $k \geq 1$, inductively defining $\operatorname{ad}^{k}(x)(y)=\operatorname{ad}(x)\left(\operatorname{ad}^{k-1}(x)(y)\right)$ for each $x, y \in \mathcal{L}$.

For a Hopf algebra $A$, let $Q(A)$ denote the module of indecomposables. Recall from [5] that whenever there is a short exact sequence $A^{\prime} \longrightarrow A \longrightarrow A^{\prime \prime}$ of homology Hopf algebras the Lie bracket $[]:, A \otimes A \longrightarrow A$ induces a left action of $A^{\prime \prime}$ on $Q\left(A^{\prime}\right)$.

Lemma 2.5 ([5, Lemma 3.12]). Let $A$ be a tensor algebra $T(V)$ and suppose that $A^{\prime \prime}$ is a free module over a tensor subalgebra $T(U)$ of $A^{\prime \prime}$. Then $Q\left(A^{\prime}\right)$ is a free $T(U)$-module.

Proposition 2.6. The commutator $[\mathcal{L}, \mathcal{L}]$ of the free Lie algebra $\mathcal{L}=$ $\mathcal{L}\langle x, u, v\rangle$ is a free Lie algebra with the following generators

$\operatorname{ad}^{k}(u)[x, x], \quad \operatorname{ad}^{k}(u)[x, u], \quad \operatorname{ad}^{k}(u)[x, v], \quad \operatorname{ad}^{k}(u)[u, v], \quad \operatorname{ad}^{k}(u)[v, v]$, $\operatorname{ad}^{k}(u)[x,[u, v]], \operatorname{ad}^{k}(u)[x,[v, v]], \operatorname{ad}^{k}(u)[v,[x, x]]$ for $k \geq 0$.

Proof. The degrees of the rank 2 commutators are different, implying that they are independent. For commutators of rank 3, because of the dimensional reason the only possible relation might hold between $[u,[x, v]]$ and $[x,[u, v]]$. But by the Jacobi identity $[u,[x, v]]=$ $[[u, x], v]+[x,[u, v]]$ and the fact that the first of these summands is non-zero, these two commutators are independent. The higher rank 
commutators are obtained by iterating the application of $\operatorname{ad}(u)$. Applying Lemma 2.5 to the tensor subalgebra $T(u) \subset S(x, u, v)$, the action of $\operatorname{ad}(u)$ is free on $W$, and therefore all the listed elements are independent. We finish the proof by comparing the given set $W$ and the Euler-Poincaré series given by Lemma 2.4.

Now we bring topology into the picture. We are aiming to the geometric realistion of the homology decomposition of $\Omega \Sigma L$ given by Lemma 2.1. For that we need to recall some preliminary definitions.

Let $p$ be a prime and $n \geq 2$. The $n$-dimensional $\bmod p$ Moore space $P^{n}(p)$ is the cofibre of the degree $p$ map on $S^{n-1}$. The $n^{t h} \bmod p$ homotopy set of a pointed space $X$ is the set of based homotopy classes

$$
\pi_{n}(X ; \mathbb{Z} / p \mathbb{Z})=\left[P^{n}(p), X\right] .
$$

The $n^{\text {th }} \bmod p$ homotopy set $\pi_{n}(X ; \mathbb{Z} / p \mathbb{Z})$ has a group structure for $n>2$ and an abelian group structure if $n>3$.

Let $G$ be a group-like space. The $\bmod p$ Samelson product is a pairing

$$
\pi_{k}(G ; \mathbb{Z} / p \mathbb{Z}) \otimes \pi_{l}(G ; \mathbb{Z} / p \mathbb{Z}) \longrightarrow \pi_{k+l}(G ; \mathbb{Z} / p \mathbb{Z})
$$

whose value at $f: P^{k}(p) \longrightarrow G$ and $g: P^{l}(p) \longrightarrow G$ is given by the composite

$$
[f, g]: P^{k+l}(p) \longrightarrow P^{k}(p) \wedge P^{l}(p) \stackrel{f \wedge g}{\longrightarrow} G \wedge G \stackrel{[,]}{\longrightarrow} G,
$$

where $[$,$] is a commutator in G$.

The Bockstein homomorphism $\beta: \pi_{n}(G ; \mathbb{Z} / p \mathbb{Z}) \longrightarrow \pi_{n-1}(G ; \mathbb{Z} / p \mathbb{Z})$ is induced by the composite

$$
P^{n-1}(p) \longrightarrow S^{n-1} \longrightarrow P^{n}(p)
$$

of the pinch map from with the inclusion. Because of the dimensional reason the composition $\beta \circ \beta$ is trivial, namely, the Bockstein homomorphism is a differential on the graded module $\pi_{*}(G ; \mathbb{Z} / p \mathbb{Z})$.

The $\bmod p$ Samelson product is compatible with the Bockstein, that is,

$$
\beta[f, g]=[\beta f, g]+(-1)^{k}[f, \beta g] .
$$

If $p \geq 5$, the $\bmod p$ Samelson product and the Bockstein operator give $\pi_{*}(G ; \mathbb{Z} / p \mathbb{Z})$ a differential graded Lie algebra structure [12].

The $\bmod p$ Whitehead product is defined by taking the adjoint of $\bmod p$ Samelson product.

There is a mod $p$ Hurewicz homomorphism defined as follows. Let $v$ be a generator of $H_{n}\left(P^{n}(p) ; \mathbb{Z} / p \mathbb{Z}\right)$ and $[l] \in \pi_{n}(X ; \mathbb{Z} / p \mathbb{Z})$. The Hurewicz homomorphism

$$
h: \pi_{n}(X ; \mathbb{Z} / p \mathbb{Z}) \longrightarrow H_{n}(X ; \mathbb{Z} / p \mathbb{Z})
$$


is defined by $h([l])=l_{*}(v)$.

Another important property of the $p$-primary Samelson product describes its behavior with respect to the Hurewicz homomorphism. As in an associative algebra, the Lie bracket in $H_{*}(G ; \mathbb{Z} / p \mathbb{Z})$ is defined by $[x, y]=x y-(-1)^{|x||y|} y x$ for $x, y \in H_{*}(G ; \mathbb{Z} / p \mathbb{Z})$. With $f$ and $g$ given as above, we have

$$
h([f, g])=[h(f), h(g)] .
$$

Hence for $p \geq 5$, the Hurewicz homomorphism is a Lie algebra homomorphism from the $p$-primary homotopy groups of $G$ to the Lie algebra of primitives in $H_{*}(G ; \mathbb{Z} / p \mathbb{Z})$.

Lemma 2.7. Each of the basis elements in Proposition [2.6 is in the image of the mod $p$ Hurewicz homomorphism in $U \mathcal{L}$ except $[x, x]$ which is spherical.

Proof. Let $\chi$ be the identity map on sphere $S^{2 n-1}$, and $\mathcal{L}(\chi)$ the Lie algebra generated by $\chi$. Then the Hurewicz homomorphism $h$ maps $\chi$ onto $x$. The Hurewicz homomorphism commutes with Samelson products and therefore maps $[\chi, \chi]$ onto $[x, x]$. Now, let $\nu$ and $\mu$ denote the identity and the Bockstein maps on $P^{2 n p-1}(p)$. Then $\beta(\nu)=\mu$ and $\beta(\mu)=0$. Let $\mathcal{L}\langle\nu, \mu\rangle$ be the free differential graded Lie algebra over $\mathbb{Z} / p \mathbb{Z}$ generated by $\{\nu, \mu\}$ with $\beta$ as a differential and the Lie structure given by the mod $p$ Whitehead product. The Hurewicz homomorphism acts as $h(\nu)=v$ and $h(\mu)=u$, where $u, v \in U \mathcal{L} \cong H_{*}(\Omega \Sigma L)$. By Formula (10), the mod $p$ Hurewicz homomorphism commutes with $\bmod p$ Whitehead products. Now the Lemma can be proved by induction on the length of the Lie bracket.

To define the desired map $\omega$ take $R$ to be the wedge of spheres and Moore spaces that correspond to the basis $W$ from Proposition 2.6 and map $\Sigma R$ by Whitehead products and $\bmod p$ Whitehead products to $\Sigma L$. More precisely, let $W=\left\{y_{i}\right\}_{i \in I} \cup z, \alpha: S^{4 n-2} \longrightarrow \Omega \Sigma L$ the Samelson product whose Hurewicz image is $z, \beta_{i}: P_{i}^{n_{i}}(p) \longrightarrow \Omega \Sigma L$ the $\bmod p$ Samelson product whose Hurewicz image is $y_{i}$ and $R=$ $S^{4 n-2} \bigvee\left(\bigvee_{i \in I} P_{i}^{n_{i}}(p)\right)$. Define $\bar{\omega}: R \longrightarrow \Omega \Sigma L$ by taking the coproduct of $\alpha$ and $\beta_{i}$ over $i \in I$. Now define the map $\omega: \Sigma R \longrightarrow \Sigma L$ as the adjoint of $\bar{\omega}$.

Proposition 2.8. In $\mathbb{Z} / p \mathbb{Z}$-homology, the induced map

$$
(\Omega \omega)_{*}: U[\mathcal{L}, \mathcal{L}] \longrightarrow U \mathcal{L}
$$

is a Hopf algebra monomorphism. 
Proof. Being an $H$-map into a homotopy associative $H$-space, $\Omega \omega$ is determined by its restriction to $R$ by the James construction. The restriction $\bar{\omega}: R \longrightarrow \Omega \Sigma L$ is, by construction, the coproduct of Samelson and $\bmod p$ Samelson products whose Hurewicz images biject onto the basis $W$ of $[\mathcal{L}, \mathcal{L}]$. Extending multiplicatively $\bar{\omega}$ to $\Omega \omega$, the homology map $(\Omega \omega)_{*}$ sends $H_{*}(\Omega \Sigma R)$ isomorphically onto the subalgebra $U[\mathcal{L}, \mathcal{L}]$ generated by Lie brackets in $H_{*}(\Omega \Sigma L)$.

2.5. The homotopy fibre of $\omega$. Denote by $F$ the homotopy fibre of $\omega$. In this section we consider the fibration sequence

$$
\Omega \Sigma R \stackrel{\Omega \omega}{\longrightarrow} \Omega \Sigma L \stackrel{\partial}{\longrightarrow} F \longrightarrow \Sigma R \stackrel{\omega}{\longrightarrow} \Sigma L
$$

and show that $F$ is homotopy equivalent to $F_{2}(n)$. Firstly, we show that $\Omega \omega$ has a left homotopy inverse. This implies that there is a homotopy decomposition of $\Omega \Sigma L$ as $\Omega \Sigma L \simeq F \times \Omega \Sigma R$, and leads towards the conclusion that $H_{*}(F) \cong S\left(x_{2 n-1}, y_{2 n p-2}, x_{2 n p-1}\right)$.

To begin, we need several of the properties of $L$ and $F_{2}(n)$. In the grand design we follow Selick's work [14] on the double suspension. On our way through it, we prove new properties of these spaces which we shall require subsequently.

Theorem 2.9 ([Selick [14]]). The spaces $F_{2}(n)$ and $L$ have the following properties:

(i) As a Hopf algebra,

$$
H_{*}\left(F_{2 k}(n)\right) \cong \bigotimes_{j=0}^{k} \Lambda\left(x_{2 n p^{j}-1}\right) \otimes \bigotimes_{j=1}^{k} \mathbb{Z} / p \mathbb{Z}\left[y_{2 n p^{j}-2}\right]
$$

with the generators primitive. The action of the Bockstein is given by $\beta\left(x_{2 n p^{j}-1}\right)=y_{2 n p^{j}-2}$

(ii) If $n>1$, then $F_{k}(n)$ is atomic for $1 \leq k \leq \infty$;

(iii) Let $K$ be the bottom 2-cell subcomplex of $L$. Then

$$
\Sigma K \wedge L \simeq S^{4 n-1} \vee S^{2(n p+n)-2} \vee P^{2(n p+n)-1}(p) \vee P^{4 n p-2}(p) ;
$$

(iv) $\Sigma^{2} L \simeq S^{2 n+1} \vee P^{2 n p+1}(p)$;

(v) $\Sigma F_{2}(n) \simeq \Sigma L \vee M$, where $M$ is a wedge of Moore spaces.

Particular emphasis should be placed upon the next property of the space $L$ since it is going to play a crucial role in proving that $F_{2}(n)$ is homotopy associative and homotopy commutative. While the next result is probably known, to author's knowledge it does not appear in the literature. 
Lemma 2.10. The space $\Sigma L \wedge L$ is homotopy equivalent to

$$
S^{4 n-1} \vee P^{2 n(p+1)-1}(p) \vee P^{2 n(p+1)-1}(p) \vee P^{4 n p-2}(p) \vee P^{4 n p-1}(p) .
$$

Proof. The reduced homology $\widetilde{H}_{*}(L)$ has three generators $x, u$ and $v$ in dimensions $2 n-1,2 n p-2$ and $2 n p-1$, respectively. The generators $u$ and $v$ are connected by the Bockstein homomorphism, that is, $\beta(v)=u$. Another way of looking at the space $L$ is by considering the cofibration sequence

$$
P^{2 n p-2}(p) \stackrel{g}{\longrightarrow} S^{2 n-1} \longrightarrow L
$$

that also defines $(2 n p-1)$-skeleton of $F_{2}(n)$. Smashing cofibration sequence (12) with $L$, there is a cofibration sequence

$$
\Sigma P^{2 n p-2}(p) \wedge L \stackrel{\Sigma g \wedge L}{\longrightarrow} \Sigma S^{2 n-1} \wedge L \longrightarrow \Sigma L \wedge L .
$$

Our aim is to show that $\Sigma g \wedge L \simeq *$.

Pinching the bottom sphere of $L$ to the based point, we get the pinch map

$$
q: L \longrightarrow P^{2 n p-1}(p)
$$

that results in the following commutative diagram

$$
\begin{gathered}
\Sigma P^{2 n p-2}(p) \wedge L \stackrel{\Sigma g \wedge L}{\longrightarrow} \Sigma S^{2 n-1} \wedge L \\
\downarrow P^{2 n p-2}(p) \wedge q \\
\Sigma P^{2 n p-2}(p) \wedge P^{2 n p-1}(p) \stackrel{\Sigma g \wedge P^{2 n p-1}(p)}{\longrightarrow} \Sigma S^{2 n-1} \wedge P^{2 n-1} \wedge q \\
\end{gathered}
$$

Since $\Sigma^{2} g \simeq \Sigma^{2} f \simeq *$, it follows that $\Sigma g \wedge P^{2 n p-1}(p) \simeq *$. Therefore our problem reduces to showing that $\gamma: \Sigma P^{2 n p-2} \wedge L \longrightarrow S^{4 n-1}$ is null homotopic. Odd sphere $S^{4 n-1}$ is an $H$-space, so it suffices to show that

$$
\Sigma \gamma: \Sigma^{2} P^{2 n p-2} \wedge L \longrightarrow \Sigma S^{4 n-1}
$$

is null homotopic. Suspending cofibration sequence (13) once more, there is a factorisation of the map $\Sigma \gamma$ through the trivial map $\Sigma^{2} g \wedge L$ :

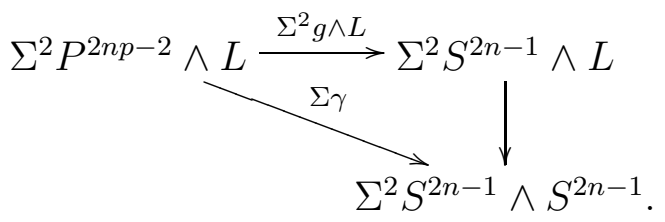

Therefore $\Sigma \gamma \simeq *$. This implies that $\gamma$ is trivial as well.

To find a left homotopy inverse of $\Omega \omega: \Omega \Sigma R \longrightarrow \Omega \Sigma L$ we require the following two lemmas, proved in [6]. 
Lemma 2.11. A map $g: \Omega X \longrightarrow Z$ has a left homotopy inverse if and only if $\Sigma g: \Sigma \Omega X \longrightarrow \Sigma Z$ has a left homotopy inverse.

Lemma 2.12. If $f: X \longrightarrow Y$ is a map between wedges of spheres and Moore spaces which induces a mod p homology isomorphism, then $f$ has a left homotopy inverse.

Lemma 2.13. The homotopy fibration $\Omega \Sigma R \stackrel{\Omega \omega}{\longrightarrow} \Omega \Sigma L \longrightarrow F$ is a trivial principal fibration, which is to say there exists a homotopy decomposition $\Omega \Sigma L \simeq F \times \Omega \Sigma R$.

Proof. To prove the Lemma it suffices to show that there is a left homotopy inverse of $\Omega \omega$, which by Lemma 2.11] exists if $\Sigma \Omega \omega$ has a left homotopy inverse. By James' theorem, $\Sigma \Omega \Sigma R \simeq \bigvee_{i=1}^{\infty} \Sigma R^{(i)}$. Applying the homotopy equivalences

$$
S^{n} \wedge S^{m} \simeq S^{m+n}, S^{m} \wedge P^{n}\left(p^{r}\right) \simeq P^{m+n}\left(p^{r}\right)
$$

and

$$
P^{m}\left(p^{r}\right) \wedge P^{n}\left(p^{r}\right) \simeq P^{m+n}\left(p^{r}\right) \vee P^{m+n-1}\left(p^{r}\right)
$$

to $\bigvee_{i=1}^{\infty} \Sigma R^{(i)}$, the space $\Sigma \Omega \Sigma R$ decomposes as a wedge of spheres and Moore spaces. Similarly, James' theorem gives $\Sigma \Omega \Sigma L \simeq \bigvee_{i=1}^{\infty} \Sigma L^{(i)}$. Using Theorem 2.9 (iv) and Lemma 2.10, there is a homotopy equivalence $\Sigma \Omega \Sigma L \simeq \Sigma L \bigvee\left(\bigvee_{\alpha} S^{n_{\alpha}}\right) \bigvee\left(\bigvee_{\beta} P^{n_{\beta}}\left(p^{r}\right)\right)$. In Proposition 2.8 we have shown that $\Omega \omega: \Omega \Sigma R \rightarrow \Omega \Sigma L$ induces a Hopf algebra monomorphism; hence $(\Sigma \Omega \omega)_{*}$ does as well. Satisfying the assumption of Lemma 2.12, the map $\Sigma \Omega \omega: \Sigma \Omega \Sigma R \longrightarrow \Sigma \Omega \Sigma L$ has a left homotopy inverse.

Corollary 2.14. As a Hopf algebra over the Steenrod algebra,

$$
H_{*}(F) \cong S\left(x_{2 n-1}, u_{2 n p-2}, v_{2 n p-1}\right) \text {. }
$$

The action of the Bockstein is given by $\beta(v)=u$, while the action of other Steenrod powers is trivial.

Proof. Consider the principal fibration $\Omega \Sigma R \stackrel{\Omega \omega}{\longrightarrow} \Omega \Sigma L \longrightarrow F$. Since $H_{*}(\Omega \Sigma L)=U \mathcal{L}$ is free $H_{*}(\Omega \Sigma R)=U[\mathcal{L}, \mathcal{L}]$-module, the EilenbergMoore spectral sequence which converges to $H_{*}(F)$ collapses. That is, $E^{2}=\operatorname{Tor}^{U[\mathcal{L}, \mathcal{L}]}(\mathbb{Z} / p, U \mathcal{L})=\mathbb{Z} / p \otimes_{U[\mathcal{L}, \mathcal{L}]} U \mathcal{L}=U \mathcal{L}_{a b}\langle x, u, v\rangle=S(x, u, v)$. Hence, $H_{*}(F) \cong S(x, u, v)$ as a coalgebra.

Use the section $s: F \longrightarrow \Omega \Sigma L$ to define the $H$-space multiplication on $F$ by the composite $F \times F \longrightarrow \Omega \Sigma L \times \Omega \Sigma L \longrightarrow \Omega \Sigma L \longrightarrow F$. Then $H_{*}(F) \cong S(x, u, v)$ as a Hopf algebra.

Finally, we want to bring $F_{2}(n)$ into the picture. Since $F_{2}(n)$ is defined as a homotopy pullback of $H$-spaces and $H$-maps, it is itself an 
$H$-space. Thus the Hopf construction can be applied to $F_{2}(n)$ inducing the following diagram of homotopy fibration sequences

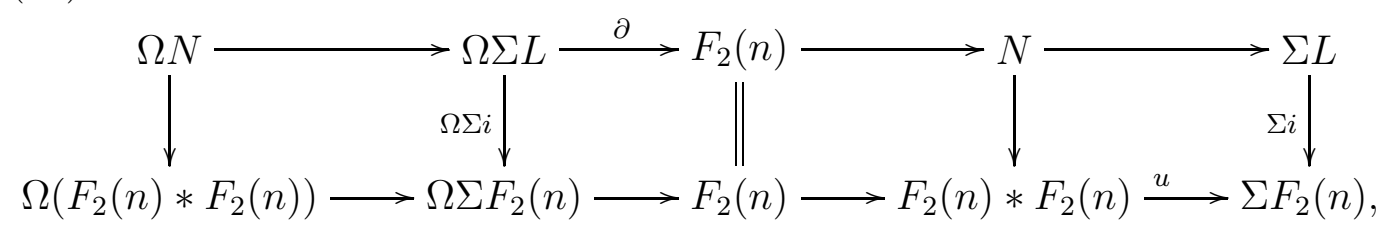

where $i: L \longrightarrow F_{2}(n)$ is the canonical inclusion of the $(2 n p-1)$ skeleton, and $N$ is the homotopy pullback of the maps $u$ and $\Sigma i$.

Lemma 2.15. The map $\Omega \Sigma i: \Omega \Sigma L \longrightarrow \Omega \Sigma F_{2}(n)$ in diagram (14) has a left homotopy inverse $s: \Omega \Sigma F_{2}(n) \longrightarrow \Omega \Sigma L$.

Proof. By Theorem 2.9 (v), the space $\Sigma L$ retracts off $\Sigma F_{2}(n)$. Therefore, $\Sigma i$ has a left homotopy inverse, and hence $\Omega \Sigma i$ does as well.

Proposition 2.16. There is a homotopy equivalence

$$
g: F_{2}(n) \longrightarrow F \text {. }
$$

Proof. Define a map $g: F_{2}(n) \longrightarrow F$ by the composite

$$
F_{2}(n) \stackrel{E}{\longrightarrow} \Omega \Sigma F_{2}(n) \stackrel{s}{\longrightarrow} \Omega \Sigma L \stackrel{\partial}{\longrightarrow} F .
$$

In homology, $E_{*}$ is the canonical inclusion of $H_{*}\left(F_{2}(n)\right)$ into $T\left(H_{*}\left(F_{2}(n)\right)\right)$; being a loop map by construction, $s$ induces an algebra map $s_{*}$; while by the construction $\partial_{*}$ is a Hopf algebra monomorphism. Therefore, $g_{*}=\partial_{*} \circ s_{*} \circ E_{*}$ is a map of algebras. By Corollary 2.14 the spaces $F_{2}(n)$ and $F$ have the same homology $S\left(x_{2 n-1}, u_{2 n p-2}, v_{2 n p-1}\right)$ as Hopf algebras over the Steenrod algebra. Since in homology $g$ induces an isomorphism in degrees $2 n-1,2 n p-2$ and $2 n p-1$, the map $g_{*}$ is an isomorphism. Thus $g$ is a homotopy equivalence.

Proof of Proposition 2.2. Identifying in homotopy fibration sequence (11) the fibre $F$ with the homotopy equivalent space $F_{2}(n)$, we obtain the fibration sequence stated by the Proposition.

\subsection{The homotopy associativity and homotopy commutativity} of $F_{2}(n)$. To begin the argument proving the homotopy associativity and homotopy commutativity of $F_{2}(n)$, we recall a pair of constructions from classical homotopy theory.

Let $X$ be a topological space, and $i_{X}$ the inclusions of $X$ into the wedge $X \vee X$. Looping, we can take the Samelson product $\left[\Omega i_{X}, \Omega i_{X}\right]$. Adjointing gives the Whitehead product $\left[\zeta_{X}, \zeta_{X}\right]$, where $\zeta_{X}=i_{X} \circ \mathrm{ev}$ 
and $e v$ is the canonical evaluation map $e v: \Sigma \Omega X \longrightarrow X$. A classical result in homotopy theory asserts that there is a homotopy fibration

$$
\Sigma \Omega X \wedge \Omega X \stackrel{\left[\zeta_{X}, \zeta_{X}\right]}{\longrightarrow} X \vee X \stackrel{i}{\longrightarrow} X \times X
$$

where $i$ is the inclusion. The universal Whitehead product of $X$ is defined as the composition

$$
\Sigma \Omega X \wedge \Omega X \stackrel{\left[\zeta_{X}, \zeta_{X}\right]}{\longrightarrow} X \vee X \stackrel{\nabla}{\longrightarrow} X
$$

where $\nabla$ is the fold map. It has the universal property that any Whitehead product on $X$ factors through $\left[\zeta_{X}, \zeta_{X}\right]$.

The linchpin in showing that $F_{2}(n)$ is homotopy associative, homotopy commutative is the following theorem proved by Theriault [16].

Theorem 2.17. Let $\Omega B \stackrel{\partial}{\longrightarrow} F \longrightarrow E \longrightarrow B$ be a homotopy fibration sequence in which $\partial$ has a right homotopy inverse. Suppose that there is a homotopy commutative diagram

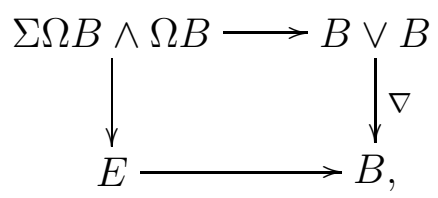

where the upper composite in the square is the universal Whitehead product of $B$. Then the multiplication on $F$ induced by the retraction off $\Omega B$ is both homotopy associative, homotopy commutative and the connecting map $\partial$ is an $H$-map.

Coming back to our case, we consider the fibration sequence

$$
\Omega \Sigma L \stackrel{\partial}{\longrightarrow} F_{2}(n) \longrightarrow \Sigma R \stackrel{\omega}{\longrightarrow} \Sigma L
$$

from Proposition 2.2. In this case the external universal Whitehead product is a map $\Psi: \Sigma \Omega \Sigma L \wedge \Omega \Sigma L \longrightarrow \Sigma L \vee \Sigma L$.

Lemma 2.18. The external universal Whitehead product

$$
\Psi: \Sigma \Omega \Sigma L \wedge \Omega \Sigma L \longrightarrow \Sigma L \vee \Sigma L
$$

is homotopic to a sum of a Whitehead product and mod $p$ Whitehead products.

Proof. Using James' theorem Theorem 2.9 (iv) and Lemma 2.10, $\Sigma \Omega \Sigma L \wedge$ $\Omega \Sigma L \simeq \Sigma M$, where $M$ is a wedge of spheres and Moore spaces. Since $\Omega \Psi$ has a left homotopy inverse, as fibration (15) splits when looped, it is an inclusion in homology. Furthermore, the Hurewicz image of each 
sphere or the mod $p$ Hurewicz image of each Moore space summand $P$ of $M$ under the composite

$$
\theta: M \stackrel{E}{\longrightarrow} \Omega \Sigma M \stackrel{\simeq}{\longrightarrow} \Omega(\Sigma \Omega \Sigma L \wedge \Omega \Sigma L) \stackrel{\Omega \Psi}{\longrightarrow} \Omega(\Sigma L \vee \Sigma L)
$$

is a bracket in $\mathcal{L}\langle V\rangle$, where $H_{*}(\Omega(\Sigma L \vee \Sigma L)) \cong H_{*}(\Omega \Sigma(L \vee L)) \cong$ $U \mathcal{L}\langle V\rangle$ for $V=\widetilde{H}_{*}(L \vee L)$. Using the identity and mod $p$ Bockstein maps on each summand of $L \vee L$, it is clear that there exists a mod $p$ Samelson product on $\Omega(L \vee L)$ which has the same Hurewicz image as $P$. Summing these mod $p$ Samelson products, one for each summand of $M$, gives a map $\lambda: M \longrightarrow \Omega(L \vee L)$ with the property that $\lambda_{*}=\theta_{*}$. Each $\bmod p$ Samelson product factors through the loop of the universal Samelson product so $\lambda$ lifts to a map $\lambda^{\prime}: M \longrightarrow \Omega(\Sigma \Omega L \wedge L)$ with $\lambda \simeq \Omega \Psi \circ \lambda^{\prime}$. Extend $\lambda^{\prime}$ to $\bar{\lambda}: \Omega \Sigma M \longrightarrow \Omega(\Sigma \Omega P \wedge \Omega P)$. As $\lambda_{*}=\theta_{*}$, we get $(\Omega \Psi \circ \bar{\lambda})_{*}=(\Omega \Psi)_{*}$. As $(\Omega \Psi)_{*}$ is a monomorphism, we must have $(\bar{\lambda})_{*}$ is an isomorphism. Hence $\bar{\lambda}$ is a homotopy equivalence. Taking adjoints then proves the Lemma.

Lemma 2.19. There is a lift

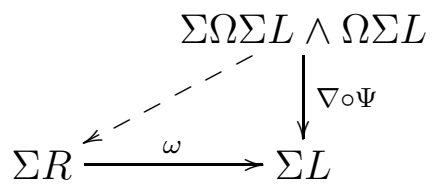

of the universal Whitehead product of $\Sigma L$ to $\Sigma R$.

Proof. Lemma 2.18 shows that the universal Whitehead product on $\Sigma L$ is homotopic to a sum of Whitehead products and mod $p$ Whitehead products. The Whitehead product defines the Lie bracket on $\Sigma L$. The set $W$ from Proposition 2.6] consists of Whitehead products and $\bmod p$ Whitehead products which form a Lie basis for $[\mathcal{L}, \mathcal{L}]$. So $\bmod p$ Whitehead products from the universal Whitehead product can be rewritten as a linear combination of basis elements.

Proposition 2.20. $F_{2}(n)$ is homotopy associative and homotopy commutative.

Proof. Applying Theorem 2.17 to the fibration sequence

$$
\Omega \Sigma L \stackrel{\partial}{\longrightarrow} F_{2}(n) \stackrel{*}{\longrightarrow} \Sigma R \stackrel{\omega}{\longrightarrow} \Sigma L
$$

and using Lemma 2.19, the Proposition follows.

2.7. A universal property of $F_{2}(n)$. In this section we show that $F_{2}(n)$ satisfies the universal property in the category of homotopy associative, homotopy commutative $H$-spaces. Let $f: L \longrightarrow Z$ be a map into a homotopy associative, homotopy commutative $H$-space. We 
want to show that there is a unique multiplicative extension $\bar{f}: F_{2}(n) \longrightarrow$ $Z$ of $f$.

Proposition 2.21. Let $h: \Omega \Sigma L \longrightarrow Z$ be an $H$-map into a homotopy commutative and homotopy associative $H$-space $Z$. Then it factors through $\partial: \Omega \Sigma L \longrightarrow F_{2}(n)$.

Proof. Denote by $g$ a right homotopy inverse of the $H$-map $\partial$ and by $e$ the homotopy equivalence $e: F_{2}(n) \stackrel{\times}{\longrightarrow} \Omega \Sigma R g \cdot \Omega \omega \Omega \Sigma L$. Define two maps $a, b: \Omega \Sigma L \longrightarrow \Omega \Sigma L$ as the composites

$$
\begin{aligned}
& a: \Omega \Sigma L \stackrel{e^{-1}}{\longrightarrow} F_{2}(n) \times \Omega \Sigma R \stackrel{\pi_{1}}{\longrightarrow} F_{2}(n) \stackrel{g}{\longrightarrow} \Omega \Sigma L \\
& b: \Omega \Sigma L \stackrel{e^{-1}}{\longrightarrow} F_{2}(n) \times \Omega \Sigma R \stackrel{\pi_{2}}{\longrightarrow} \Omega \Sigma R \stackrel{\Omega \omega}{\longrightarrow} \Omega \Sigma L .
\end{aligned}
$$

In the following diagram

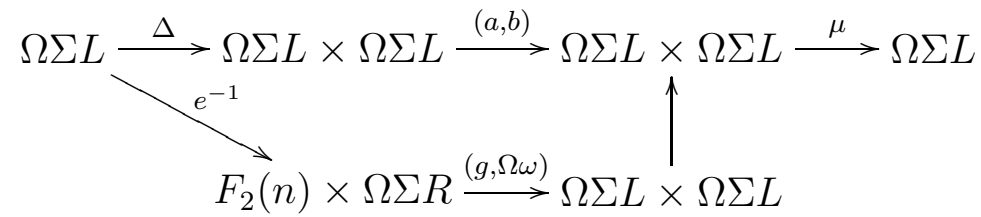

the top row composition is $a+b$, while the bottom row is the identity on $\Omega \Sigma L$. The commutativity of the diagram gives $\operatorname{Id}_{\Omega \Sigma L} \simeq a+b$.

Being an $H$-map, $h$ is determined by its restrictions on the both of the factors of $\Omega \Sigma L$, that is, $h \simeq h \circ \operatorname{Id}_{\Omega \Sigma L} \simeq h \circ(a+b) \simeq h \circ a+h \circ b$.

Showing that the composite

$$
\Omega \Sigma R \stackrel{\Omega \omega}{\longrightarrow} \Omega \Sigma L \stackrel{h}{\longrightarrow} Z
$$

is null homotopic, we have $h \circ b \simeq *$ and hence $h \simeq h \circ a$ that proves the Proposition.

As a composite of $H$-maps, $h \circ \Omega \omega$ is itself an $H$-map. Therefore by the James construction, it is uniquely determined by its restriction to $R$. The composite

$$
R \stackrel{E}{\longrightarrow} \Omega \Sigma R \stackrel{\Omega \omega}{\longrightarrow} \Omega \Sigma L
$$

is a wedge sum of Samelson products on $R$ as it is the adjoint of a wedge sum of Whitehead products $\omega: \Sigma R \longrightarrow \Sigma L$. Being an $H$-map, $h$ preserves Samelson products. Therefore the wedge of Samelson products $\Omega \omega \circ E$ on $R$ composed with $h$ into the homotopy commutative $H$-space $Z$ is trivial.

Consider the fibration sequence

$$
\Omega \Sigma L \stackrel{\partial}{\longrightarrow} F_{2}(n) \stackrel{*}{\longrightarrow} \Sigma R \stackrel{\omega}{\longrightarrow} \Sigma L .
$$


Define a map $\bar{f}$ as the composite

$$
\bar{f}: F_{2}(n) \stackrel{g}{\longrightarrow} \Omega \Sigma L \stackrel{\tilde{f}}{\longrightarrow} Z,
$$

where $g: F_{2}(n) \longrightarrow \Omega \Sigma L$ is a right homotopy inverse of the map $\partial: \Omega \Sigma L \longrightarrow F_{2}(n)$, and $\tilde{f}: \Omega \Sigma L \longrightarrow Z$ is the canonical extension of $f$ given by the James construction.

Theorem 2.22. Let $Z$ be a homotopy associative, homotopy commutative $H$-space, and $f: L \longrightarrow Z$ a given map. Then there exists an extension to a unique up to homotopy $H-\operatorname{map} \bar{f}: F_{2}(n) \longrightarrow Z$.

Proof. Let us consider the map $\bar{f}$ given by

$$
\bar{f}: F_{2}(n) \stackrel{g}{\longrightarrow} \Omega \Sigma L \stackrel{\tilde{f}}{\longrightarrow} Z,
$$

as a candidate for the multiplicative extension of $f$. Notice that there is a commutative diagram

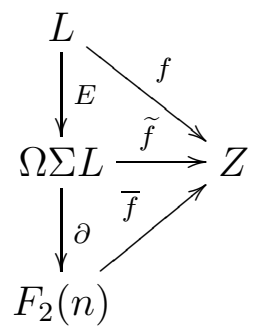

since the upper triangle commutes by the James construction, while the commutativity of the lower diagram is given by Proposition 2.21] Diagram (16) ensures that $\bar{f}$ is an extension of $f$.

Now, we shall show that $\bar{f}$ is an $H$-map by showing that the diagram

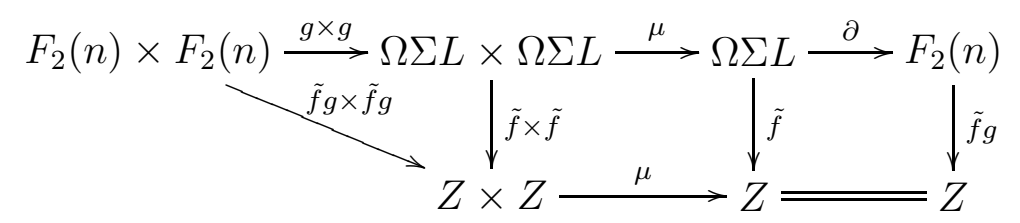

commutes. The left triangle commutes by definition; the middle square commutes since $\tilde{f}$ is an $H$-map; and the commutativity of the right square is given by Proposition 2.21. Summing this up, diagram (17) commutes.

Finally we are left to show that $\bar{f}=\tilde{f} \circ g: F_{2}(n) \longrightarrow Z$ is the unique $H$-map extending $f: L \longrightarrow Z$. To prove that we use the uniqueness of $\tilde{f}$ asserted by the James construction and the result of Theorem 2.17 which shows that the connecting map $\partial: \Omega \Sigma L \longrightarrow F_{2}(n)$ is an $H$-map. 
Let $\bar{f}, \bar{g}$ be two extensions $F_{2}(n) \stackrel{\bar{f}, \bar{g}}{\longrightarrow} Z$ of the map $f: L \longrightarrow Z$ which are $H$-maps. Precompose both maps with the $H$-map $\partial: \Omega \Sigma L \longrightarrow$ $F_{2}(n)$. We obtain two multiplicative extensions $\Omega \Sigma L \stackrel{\partial}{\longrightarrow} F_{2}(n) \stackrel{\bar{f}, \bar{l}}{\longrightarrow} Z$ of $f: L \longrightarrow Z$. By the uniqueness of an $H$-map $\Omega \Sigma L \longrightarrow Z$ extending $f$ in the James construction, it follows that $\bar{f} \circ \partial \simeq \bar{l} \circ \partial$. Precomposing both compositions with the right homotopy inverse $g: F_{2}(n) \longrightarrow \Omega \Sigma L$ of the map $\partial$, we get

$$
\bar{f} \circ \partial \circ g \simeq \bar{l} \circ \partial \circ g
$$

Hence

$$
\bar{f} \simeq \bar{l}
$$

and the uniqueness assertion is proved. This finishes the proof of the Theorem.

The following theorem summarises Proposition 2.20 and Theorem 2.22

Theorem 2.23. $F_{2}(n)$ is a universal space of $L$.

The uniqueness assertion of the theorem is powerful. It can be used to show that two $H$-maps from $F_{2}(n)$ to a homotopy associative, homotopy commutative $H$-space are homotopic by comparing their restriction to $L$. This property is the foundation stone in the application we consider in the next section.

\section{The $d_{1}$-Differential in the EHP SPECtral SEquence}

3.1. Extension of a formula for the $d_{1}$-differential to $F_{2}(n p)$. The problem we want to discuss in this section is that of calculating the unstable homotopy groups of spheres using the $E H P$ spectral sequence and applying the universality of $F_{2}(n)$.

Recall from the Introduction that the $d_{1}$-differential in the $E H P$ spectral sequence is induced by the composition $\Omega^{3} S^{2 n p+1} \stackrel{\Omega P}{\longrightarrow} \Omega J_{p-1}\left(S^{2 n}\right) \stackrel{H}{\longrightarrow}$ $\Omega S^{2 n p-1}$. In [8] Gray constructed a map $\varphi_{n}: \Omega^{2} S^{2 n p+1} \longrightarrow S^{2 n p-1}$ with the property that $\Omega \varphi_{n}=H \circ \Omega P$. Therefore, the $d_{1}$-differential can be considered as $d_{1}(x)=\varphi_{n} \cdot x$. Using the existence of the map $\varphi_{n}$ and the universality of $\Omega J_{p-1}\left(S^{2 n}\right)$ when $p \geq 3$, Gray [9] developed a formula for $d_{1}$ applicable to elements which are not double suspensions.

One would like to have a formula for the $d_{1}$-differential applicable to its whole domain. Since $F_{2}(n)$ is a third approximation of the double suspension, after $S^{2 n-1}$ and $\Omega J_{p-1}\left(S^{2 n}\right)$, we would like to extend the description of $d_{1}$ from $\Omega J_{p-1}\left(S^{2 n p}\right)$ to $F_{2}(n p)$. 
There are a few points which should be commented upon. Recall that $W_{n}$ denotes the homotopy fibre of the double suspension $E^{2}: S^{2 n-1} \longrightarrow$ $\Omega^{2} S^{2 n+1}$. It has been shown in [10] that $W_{n}$ is a loop space, namely that there exists a fibration sequence

$$
W_{n} \longrightarrow S^{2 n-1} \stackrel{E^{2}}{\longrightarrow} \Omega^{2} S^{2 n+1} \longrightarrow B W_{n}
$$

where $B W_{n}$ is defined as the homotopy fibre of $\varphi_{n}$,

$$
B W_{n} \longrightarrow \Omega^{2} S^{2 n p+1} \stackrel{\varphi_{n}}{\longrightarrow} S^{2 n p-1} .
$$

The following lemma states the existence of two fibration sequences involving $F_{2}(n)$ which are analogous to the two classical EHP fibrations.

Lemma 3.1. There exist homotopy fibration sequences

$$
W_{n p} \stackrel{P}{\longrightarrow} F_{2}(n) \stackrel{E}{\longrightarrow} \Omega^{2} S^{2 n+1} \stackrel{H}{\longrightarrow} B W_{n p}
$$

and

$$
\Omega S^{2 n p-1}\{p\} \stackrel{P}{\longrightarrow} S^{2 n-1} \stackrel{E}{\longrightarrow} F_{2}(n) \stackrel{H}{\longrightarrow} S^{2 n p-1}\{p\} .
$$

Proof. The homotopy pullback defining $F_{2}(n)$ can be extended to the homotopy pullback diagram

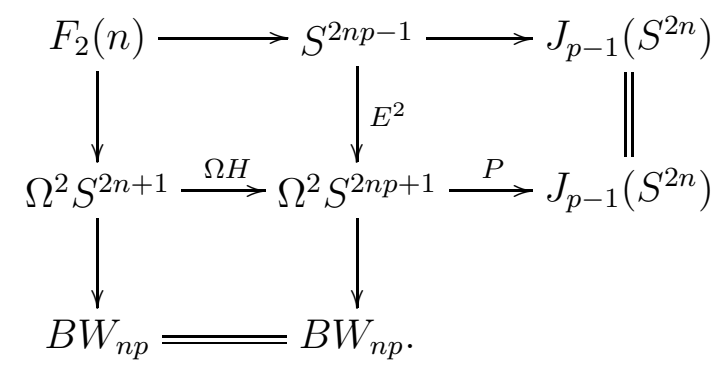

of homotopy fibration sequences. The left column of the diagram is the first fibration sequence whose existence is claimed in the Lemma.

The second homotopy fibration sequence of the Lemma is obtained from the following homotopy pullback

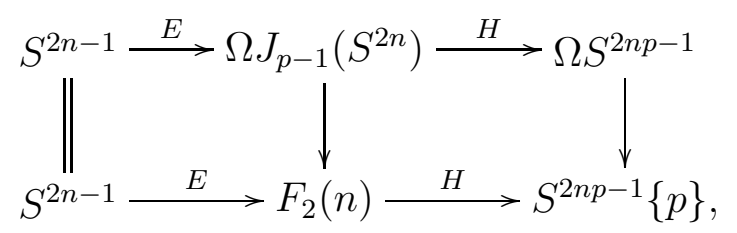

whose existence is proved by Selick in [14, Theorem 10]. 
In our programme for finding a formula for the $d_{1}$-differential we consider the map

$$
\alpha: F_{2}(n p) \stackrel{E}{\longrightarrow} \Omega^{2} S^{2 n p+1} \stackrel{\varphi_{n}}{\longrightarrow} S^{2 n p-1} \stackrel{E}{\longrightarrow} F_{2}(n p) .
$$

Recall that $L_{n p}$ is the $\left(2 n p^{2}-1\right)$-skeleton of $F_{2}(n p)$. The space $F_{2}(n p)$ is an $H$-space, so it has a $p$-power map.

Proposition 3.2. $\quad$ (i) The restriction of

$$
\alpha: F_{2}(n p) \stackrel{E}{\longrightarrow} \Omega^{2} S^{2 n p+1} \stackrel{\varphi_{n}}{\longrightarrow} S^{2 n p-1} \stackrel{E}{\longrightarrow} F_{2}(n p)
$$

to $L_{n p}$ is homotopic to the restriction of the p-power map to $L_{n p}$.

(ii) If $\alpha$ is an $H$-map, then $\alpha \simeq p$.

Proof. Look at the difference $D$ of $\alpha$ and the $p$-power map restricted to the $\left(2 n p^{2}-1\right)$-skeleton of $F_{2}(n p)$. Since $\left(F_{2}(n p)\right)_{\left(2 n p^{2}-1\right)}$ is homotopy equivalent to the $\left(2 n p^{2}-1\right)$-skeleton of $\Omega^{2} S^{2 n p+1}$ the map $\alpha$ restricted to $\left(F_{2}(n p)\right)_{\left(2 n p^{2}-1\right)}$ is homotopic to the composite

$$
\widetilde{\alpha}:\left(\Omega^{2} S^{2 n p+1}\right)_{\left(2 n p^{2}-1\right)} \stackrel{\varphi_{n}}{\longrightarrow} S^{2 n p-1} \stackrel{E}{\longrightarrow} F_{2}(n p) .
$$

Theriault has proved [17] that $E^{2} \circ \varphi_{n} \simeq p$. Hence the difference between $E \circ \alpha$ and the $p$ power map is null homotopic. Therefore $D$ factors through $W_{n p^{2}}$, the homotopy fibre of $E: F_{2}(n p) \longrightarrow \Omega^{2} S^{2 n p+1}$. Applying the homology Serre spectral sequence to the fibration sequence $W_{n p^{2}} \longrightarrow F_{2}(n p) \stackrel{E}{\longrightarrow} \Omega^{2} S^{2 n p+1}$, we have $W_{n p^{2}}$ is $2 n p^{3}-4$ connected, and therefore $D$ is null homotopic.

The second assertion of the Proposition follows from part (i) since $F_{2}(n p)$ is the universal space of $L_{n p}$. Namely, all relevant maps are $H$-maps and their composite is uniquely determined by the restriction to $L_{n p}$.

The following Theorem gives the main result, which is obtained by applying the universality of $F_{2}(n)$ to the EHP spectral sequence calculation of the unstable homotopy groups of spheres.

Theorem 3.3. The composite

$$
F_{2}(n p) \stackrel{E}{\longrightarrow} \Omega^{2} S^{2 n p+1} \stackrel{\varphi_{n}}{\longrightarrow} S^{2 n p-1} \stackrel{E}{\longrightarrow} F_{2}(n p)
$$

is the p-power map if either

(i) $x \in \pi_{*}\left(F_{2}(n p)\right)$ is an element which is a lift through $H: F_{2}(n p) \longrightarrow S^{2 n p^{2}-1}\{p\}$ of an element in the image of $E: P^{2 n p^{2}-2}(p) \longrightarrow \Omega S^{2 n p^{2}-1}\{p\}$

or

(ii) the map $\alpha$ is an $H$-map. 
Proof. First we show that if $H(x)=E(u)$, then $x \in \operatorname{Im}\left(\pi_{*}\left(L_{n p}\right) \longrightarrow\right.$ $\pi_{*}\left(F_{2}(n p)\right)$. The assumptions can be presented in the form of the diagram

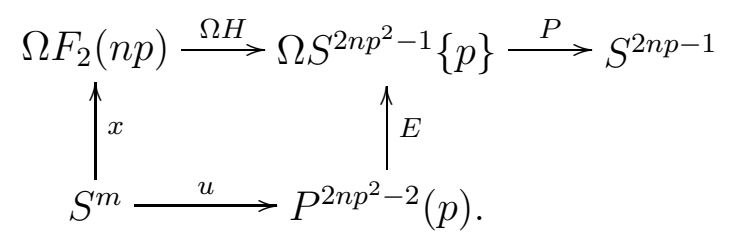

Let $\omega_{n p}$ be the composite $P^{2 n p^{2}-2}(p) \stackrel{E}{\longrightarrow} \Omega S^{2 n p^{2}-1}\{p\} \stackrel{P}{\longrightarrow} S^{2 n p-1}$. Then

$$
\omega_{n p} \circ u \simeq 0
$$

since it factors through two consecutive terms of the homotopy fibration sequence

$$
\Omega F_{2}(n p) \stackrel{\Omega H}{\longrightarrow} \Omega S^{2 n p^{2}-1}\{p\} \stackrel{P}{\longrightarrow} S^{2 n p-1} .
$$

Therefore $u$ factors through the fibre $F_{\omega_{n p}}$ of $\omega_{n p}$. Putting this all together we obtain the following diagram

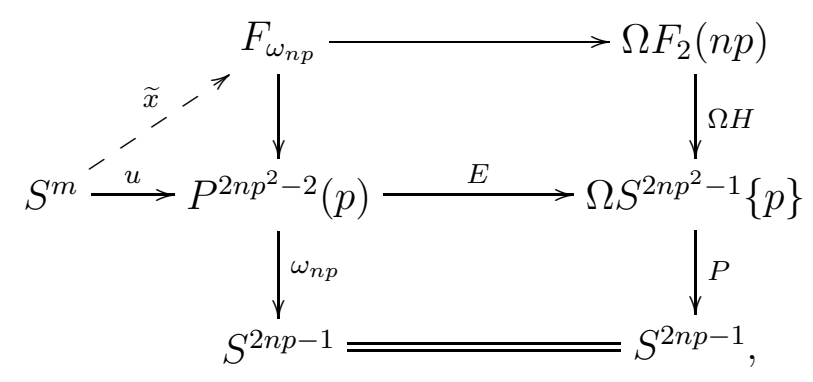

where $\widetilde{x}$ is a lift of $u$ to $F_{\omega_{n p}}$.

It follows from the defining property of a pullback that the map $x$ factors as

$$
x: S^{m} \stackrel{\widetilde{x}}{\longrightarrow} F_{\omega_{n p}} \longrightarrow \Omega F_{2}(n p) .
$$

We claim that the map $F_{\omega_{n p}} \longrightarrow \Omega F_{2}(n p)$ factors through $\Omega L_{n p}$. To see this, let $X$ be the fibre of the pinch map $q: L_{n p} \longrightarrow P^{2 n p^{2}-1}(p)$ to the top Moore space. Then there is a fibration sequence

$$
\Omega P^{2 n p^{2}-1}(p) \longrightarrow X \longrightarrow L_{n p} \longrightarrow P^{2 n p^{2}-1}(p) .
$$

Starting with the diagram

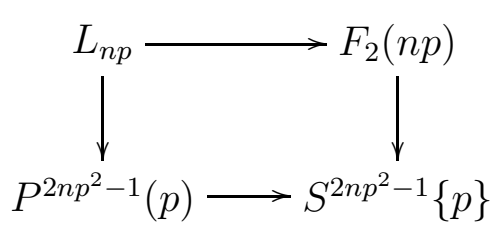


that includes the top Moore space of $L_{n p}$ into $S^{2 n p^{2}-1}\{p\}$, there is an induced map of homotopy fibrations

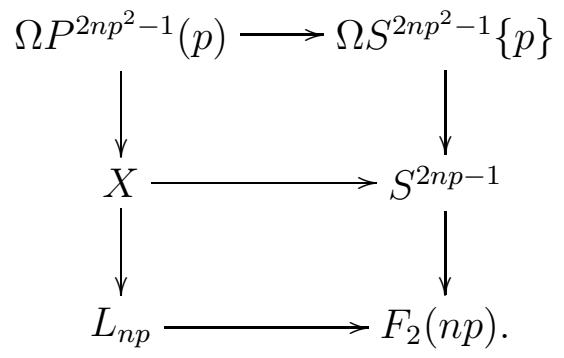

Including the bottom cell $S^{2 n p-1}$ into $X$, let us consider the composition

$$
f: P^{2 n p^{2}-2}(p) \stackrel{\omega_{n p}}{\longrightarrow} S^{2 n p-1} \stackrel{i}{\longrightarrow} X \longrightarrow L_{n p} .
$$

By looking at the map

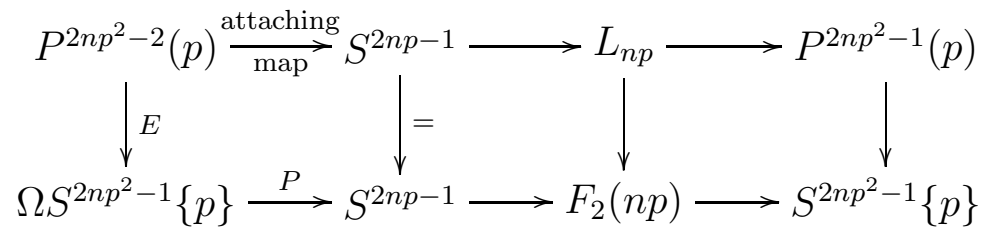

between a cofibration homotopy sequence and a fibration sequence, we conclude that the map $\omega_{n p}$ is homotopic to the attaching map defining $L_{n p}$. Thus the map $f$ is null homotopic since it factors through the cofibration sequence

$$
P^{2 n p^{2}-2}(p) \longrightarrow S^{2 n p-1} \longrightarrow L_{n p}
$$

Therefore, there exists a lift $E: P^{2 n p^{2}-2}(p) \longrightarrow \Omega P^{2 n p^{2}-1}(p)$ closing the diagram

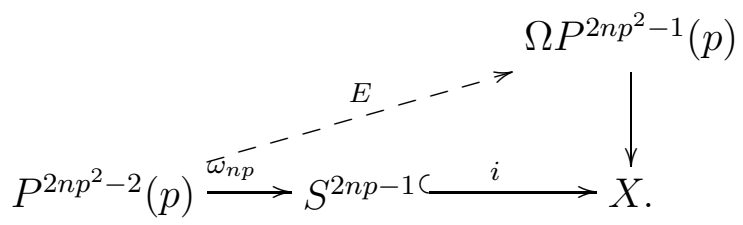


This lift allows us to take the juxtaposition of the two fibration diagrams

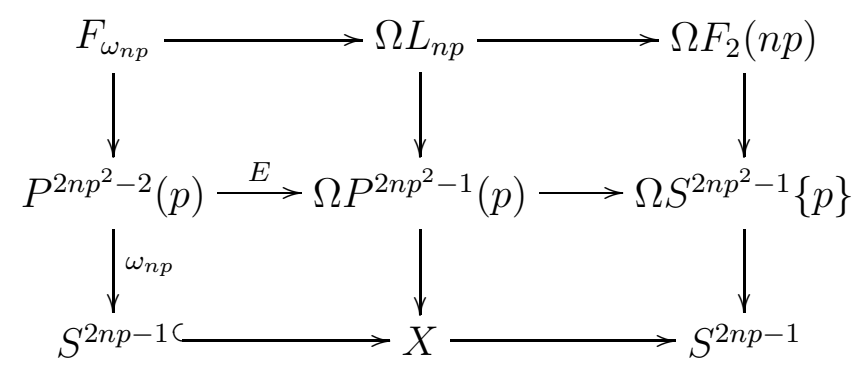

Diagram (18) shows that there is a factorisation of the map $F_{\omega_{n p}} \longrightarrow$ $\Omega F_{2}(n p)$ through $\Omega L_{n p}$. Now both statements of the Theorem follow from Proposition 3.2 .

Remark 3.1. Passing to the homotopy groups under the assumptions of Theorem 3.3 the formula for the $d_{1}$-differential takes the form

$$
E d_{1}(E x)=p \cdot x .
$$

\subsection{Partial results.}

Proposition 3.4. Restricted to the $\left(2 n p^{3}-4\right)$-skeleton the composite

$$
F_{2}(n p) \longrightarrow \Omega^{2} S^{2 n p+1} \stackrel{\varphi_{n}}{\longrightarrow} S^{2 n p-1} \stackrel{E}{\longrightarrow} F_{2}(n p)
$$

is homotopic to the p-power map restricted to $\left(F_{2}(n p)\right)_{\left(2 n p^{3}-4\right)}$.

Proof. First let us notice that $\left(F_{2}(n p)\right)_{\left(2 n p^{3}-3\right)}$ is homotopy equivalent to the $\left(2 n p^{3}-3\right)$-skeleton of $\Omega^{2} S^{2 n p+1}$. Therefore, the map

$$
\bar{\alpha}:\left(\Omega^{2} S^{2 n+1}\right)_{\left(2 n p^{3}-4\right)} \stackrel{\varphi_{n}}{\longrightarrow} S^{2 n p-1} \stackrel{E}{\longrightarrow} F_{2}(n p)
$$

is homotopic to the restriction of

$$
\alpha: F_{2}(n p) \stackrel{E}{\longrightarrow} \Omega^{2} S^{2 n p+1} \stackrel{\varphi_{n}}{\longrightarrow} S^{2 n p-1} \stackrel{E}{\longrightarrow} F_{2}(n p)
$$

to the $\left(2 n p^{3}-3\right)$-skeleton of $F_{2}(n p)$. Theriault has proved [17] that $E^{2} \circ \varphi_{n} \simeq p$. Hence the difference $D$ between $E \circ \alpha$ and the $p$-power map is null homotopic. Therefore $D$ factors through $W_{n p^{2}}$, the homotopy fibre of $E: F_{2}(n p) \longrightarrow \Omega^{2} S^{2 n p+1}$. Since $W_{n p^{2}}$ is $2 n p^{3}-4$ connected, $D$ is null homotopic.

Corollary 3.5. Formula (19) for the $d_{1}$-differential in the EHP-spectral sequence is correct up to the $\left(2 n p^{3}-4\right)$-skeleton of $\Omega^{2} S^{2 n p+1}$.

Proof. It follows immediately from Theorem 3.3 (ii) since restricted to the given skeleton of $\Omega^{2} S^{2 n p+1}$ the map $\alpha$ is an $H$-map. 


\section{REFERENCES}

1. BibliographyF. Adams. The sphere, considered as an $H$-space $\bmod p$. Quart. J. Math. Oxford. Ser. (2). 12 (1961), 52-60.

2. BibliographyD. Anick. Differential algebras in topology. Research Notes in Mathematics 3. (A K Peters Ltd, Wellesley, MA, 1993).

3. BibliographyD. Anick and B. Gray. Small $H$ spaces related to Moore spaces. Topology 34 (1995), 859-881.

4. BibliographyF. R. Cohen, T. J, Lada, and P. J. May. The homology of iterated loop spaces. Lecture Notes in Mathematics Vol. 533 Springer-Verlag. (1976).

5. BibliographyF. R. Cohen, J. C. Moore and J. A. Neisendorfer. Torsion in Homotopy Groups. Annals of Math. 109 (1979), 121-168.

6. BibliographyF. R. Cohen, J. C. Moore and J. A. Neisendorfer. Exponents in homotopy theory. Algebraic Topology and Algebraic K-theory. (ed W. Browder). Annals of Math. Study. 113 (Princeton University Press, Princeton, 1987), 334 .

7. BibliographyA. Dold and R. Thom. Une généralisation de la notion d'espace fibré. Application aux produits symétriques infinis. C. R. Acad. Sci. Paris 242 (1956), 1680-1682.

8. BibliographyB. Gray. On the double suspension. Algebraic topology (Arcata, CA, 1986). Lecture Notes in Math. 1370 (Springer, Berlin 1989), 150-162.

9. BibliographyB. Gray. Homotopy commutativity and the EHP sequence. Proc. Internat. Conf., 1988. Contemp. Math. 96 (Amer. Math. Soc., Providence, RI, 1989), 181-188.

10. BibliographyB. Gray. On the iterated suspension. Topology 27 (1988), 301-310.

11. BibliographyJ. Grbić. Universal spaces in the category of homotopy associative, homotopy commutative $H$-spaces. PhD thesis. University of Aberdeen (2004).

12. BibliographyJ. Neisendorfer. Primary homotopy theory. Mem. Amer. Math. Soc. 25 (1980).

13. BibliographyD. Ravenel. Complex cobordism and stable homotopy groups of spheres. Pure and Applied Mathematics. 121 (1986).

14. BibliographyP. Selick. A spectral sequence concerning the double suspension. Invent. Math. 64 (1981), 15-24.

15. BibliographyJ.P. Serre. Homologie singulière des espaces fibrés. Applications. Ann. of Math. (2). 54 (1951), 425-505.

16. BibliographyS. D. Theriault. Properties of Anick's spaces. Trans. Amer. Math. Soc. 353 (2001), 1009-1037.

17. BibliographyS. D. Theriault. Proofs of two conjectures of Gray involving the double suspension. Proc. Amer. Math. Soc. 131 (2003), 2953-2962.

18. BibliographyH. Toda. On the double suspension $E^{2}$. J. Inst. Polytech. Osaka City Univ. Ser. A. 7 (1956), 103-145.

19. BibliographyH. Toda. Composition methods in homotopy groups of spheres. Annals of Mathematics Studies 49 (Princeton University Press, Princeton, 1962). 